\title{
Aplicação da Distribuição de Probabilidade de Weibull nos Estudos Eólicos de Pernambuco
}

\author{
Marinho, M. H. N. \\ Escola Politécnica de Pernambuco \\ Universidade de Pernambuco \\ 50.720-001 - Recife, Brasil \\ marinho75@poli.br
}

\author{
Siqueira, R. N. \\ Escola Politécnica de Pernambuco \\ Universidade de Pernambuco \\ 50.720-001 - Recife, Brasil \\ renatosiqueira@hotmail.com
}

\begin{abstract}
Resumo Neste trabalho, o objetivo foi estudar o potencial eólico de Pernambuco, para tal exploração, utilizouse neste estudo a melhor distribuição estatística aplicada às ocorrências do vento na região Nordeste do Brasil, que tem sido apontada como a função de densidade de probabilidade de Weibull. Os dados de velocidade de vento (2005 a 2010) e das vazões afluentes (1931 a 2010) foram obtidos junto ao Instituto Nacional de Meteorologia - INMET/ $3^{\circ}$ Distrito de Meteorologia, e ao Operador Nacional do Sistema Elétrico - ONS. Foi feito um comparativo com um periodo de cheia e com um período de seca dos rios, mostrando que há uma variação significativa do periodo de cheia para um período de seca, na energia eólica gerada, ambos os comparativos são feitos com a vazão afluente da Usina Hidrelétrica de Sobradinho. Comprovou-se neste estudo hipotético que a variação de energia eólica gerada é mínima, pois a geração eólica no período de seca foi de aproximadamente $269 \mathrm{MWh} / \mathrm{h}$ e no periodo de cheia foi de $264 \mathrm{MWh} / \mathrm{h}$.
\end{abstract}

Palavras-Chave: Complementaridade; Energia Eólica; Regime Hidrológico; Geração Hidroeólica; Distribuição de Probabilidade de Weibull

\begin{abstract}
In this work, the objective was to study the wind potential of Pernambuco, to such exploitation, was used in this study the best statistical distribution applied to instances of the wind in the Northeast region of Brazil, which has been identified as the probability density function of Weibull. The data of wind speed ( 2005-2010) and inflows (1931-2010) were obtained from the National Institute of Meteorology - INMET / 3rd District of Meteorology and the National Electric System Operator - ONS . A comparison was made with a full period and a period of dry rivers, showing that there is a significant variation of the full period for a period of drought, wind power generated, both comparisons are made with the inflow of Plant hydroelectric Sobradinho. It was proved in this case study that the variation of wind power generated is minimal since the wind generation during the dry period was approximately $269 \mathrm{MWh} / \mathrm{h}$ during the flooding was $264 \mathrm{MWh} / \mathrm{h}$.
\end{abstract}

Keywords: Complementary, Wind Energy, Hydrogical Regime; Generation Hidroeólica; Weibull Probability Distrubution. 


\section{Introdução}

A energia armazenada nos reservatórios das usinas hidrelétricas para o planejamento de longo e médio prazo é discretizada mensal ou semanalmente nos horizontes de estudo e apresenta variações, devido à disponibilidade hídrica natural, apresenta flutuações sazonais com amplitude significativa. Os períodos secos requerem estratégias de otimização da gestão dos reservatórios para evitar racionamentos de energia com fontes de energia renováveis (MARINHO, 2012).

A energia eólica pode contribuir de forma relevante para resolver o grande dilema do uso da água dos rios nordestinos (água para gerar eletricidade versus água para irrigação/abastecimento), pois se percebe que as maiores velocidades de vento no nordeste do Brasil ocorrem justamente quando o fluxo de água dos rios é mínimo, foi feito um comparativo com um período de cheia e com um período de seca, mostrando que há uma variação significativa do período de cheia para um período de seca na variação de energia eólica gerada, ambos os comparativos são feitos com a vazão afluente da Usina Hidrelétrica de Sobradinho. As centrais eólicas hipotéticas neste estudo foram Triunfo, Surubim, Recife, Ouricuri, Pesqueira, Petrolina, Garanhuns, Cabrobó e Arcoverde, instaladas no estado de Pernambuco no nordeste do Brasil, elas poderão produzir grandes quantidades de energia elétrica evitando que se tenha que utilizar a água dos rios, principalmente no período de seca.

Os dados de velocidade de vento de 2005 a 2012 das estações meteorológicas foram obtidos junto ao Instituto Nacional de Meteorologia - INMET/ $3^{\circ}$ Distritos de Meteorologia - DISME. Utilizaram-se as vazões afluentes médias mensais da série histórica de 74 anos (1931 a 2010) do posto hidrológico da usina hidrelétrica de Sobradinho.

Na tentativa estudar o potencial eólico de Pernambuco, foi escolhida a distribuição de probabilidade de Weibull, segundo MARINHO et al. (2006) e MARINHO e AQUINO (2009) que comprovaram com estudos hipotéticos. Comparando-a com a distribuição Rayleigh também utilizada em estudos eólicos, segundo Bernardo et al. (2002), Na Arábia Saudita, Sahin \& Aksakal (1998) usaram as distribuições de Weibull e Rayleight para avaliar o desempenho de um sistema eólico e concluíram que os resultados alcançados com os dados experimentais se aproximaram melhor daqueles gerados com o modelo de Weibull, com erro relativo de apenas 3,46\%.

A partir dos dados de velocidade de vento se obteve a velocidade de vento para torres instaladas hipoteticamente nas 9 (nove) centrais eólicas citadas, a 85, 98 e 120 metros de altura. Para as devidas simulações foi utilizada a curva de potência do aerogerador da ENERCON E-92 2.3 MW.
Estas etapas de simulação foram realizadas com o auxilio de ferramentas computacionais, tais como: MATLAB (2010) e Microsoft Office Excel 2007.

No presente trabalho foram desenvolvidos cálculos utilizando a distribuição de Weibull que modelam a velocidade de vento de acordo com as características locais, essa modelagem depende de dois parâmetros que são $\mathrm{k}$ (de forma) e c (de escala), assim possibilitando obter a energia eólica gerada hipoteticamente para as alturas estabelecidas. Desta forma, é possível utilizar os resultados destas previsões para programar soluções para os períodos secos que requerem estratégias de otimização da gestão dos reservatórios, utilizando a vazão economizada pela geração eólica principalmente em períodos de seca, para que se possam evitar como, por exemplo, racionamentos.

\section{Distribuição de Probabilidade de Weibull nos Estudos Eólicos}

As primeiras aplicações da distribuição de Weibull em estudos eólicos se restringiam à representação da distribuição de velocidades em análises dos carregamentos de vento [Danvenport, 1963]. Treze anos após, foi constatado que para a maioria das ocorrências de vento a distribuição de Weibull fornece um bom ajuste [Justus et al., 1976] [Hennessey, 1977].

Em particular, Hennessey examinou as propriedades da distribuição de Weibull em seus detalhes e constatou que existe uma forte relação entre os momentos da velocidade de vento e os parâmetros k e c. Notou, ainda, que os mínimos parâmetros estatísticos requeridos para se estimar o potencial energético do vento são a velocidade média e o desvio padrão da série temporal.

\subsection{Estilos}

A função densidade de probabilidade do método de Weibull é um caso especial da distribuição de Pearson tipo III ou da função de distribuição de Gama generalizada com dois parâmetros. Se uma distribuição de vento pode ser representada pela função de densidade de probabilidade de Weibull, ROHATGI e NELSON (1994), ela então é regida pela seguinte equação:

$$
f(v)=\frac{k}{c}\left(\frac{v}{c}\right)^{k-1} e^{-\left(\frac{v}{c}\right)^{k}}
$$

Onde $k>0, c>0$ e $v>0$ em que $k$ é o parâmetro de fator de forma, $c$ é chamado de fator de escala e $v$ é a velocidade de vento. A função do MS Excel 2007 que representa Eq.(1) é: =WEIBULL(x, alfa, beta, cumulativo), onde $\mathbf{x}$ é a velocidade $\mathrm{v}$, alfa é $\mathrm{k}$ e beta $\mathrm{c}$, cumulativo é um valor booleano que pode ser FALSE (falso) que se 
refere a Eq.(1) ou TRUE (verdadeiro) que se refere a distribuição cumulativa Weibull que não é utilizada no presente trabalho.

O valor médio das velocidades dos ventos de um local com as características de Weibull é expresso pela seguinte equação:

$$
\bar{v}=\int_{0}^{\infty} v \cdot \frac{k}{c} \cdot\left(\frac{v}{c}\right)^{k-1} \cdot e^{-\left(\frac{v}{c}\right)^{k}} d v
$$

em que se usando a função gama, o valor médio da velocidade de vento pode ser encontrado de modo simplificado através da equação:

$$
\bar{v}=c \Gamma\left(1+\frac{1}{k}\right)
$$

em que $\Gamma$ é função gama.

Existem diversos métodos disponíveis para se determinar os valores dos parâmetros de $\mathrm{k}$ e $\mathrm{c}$ a partir dos dados de vento medidos em um local.

Os parâmetros de distribuição de Weibull foram calculados utilizando o Método da Velocidade Média e Desvio Padrão. Considerando os valores da velocidade média, calculada para as alturas de 85,98 e 120 metros de altura, a partir dos dados de velocidade de vento de 2005 a 2012 das estações meteorológicas medidas à 10 metros de altura de 9 (nove) estações anemométricas, pela equação empírica GIPE (1993) e LYSEN (1982):

$$
v(h)=v_{1}\left(\frac{h}{h_{1}}\right)^{\alpha}
$$

em que:

$v_{1}$ - velocidade na altura $h_{1}$ (conhecida),

$v(h)$ - velocidade na altura $\mathrm{h}$,

$\mathrm{h}$ - altura para velocidade $v(h)$,

$h_{1}$ - altura da velocidade $v_{1}$,

$\alpha$-coeficiente dependente da natureza do terreno.

O desvio padrão foi obtido pela função do MS Excel 2007 =DESVPAD(núm1;núm2,...), a partir dos dados de velocidade de vento de 2005 a 2012 das estações meteorológicas medidas a 10 metros de altura de 9 (nove) estações anemométricas.

Considerando os valores da velocidade média e desvio padrão. $\mathrm{O}$ valor de $\mathrm{k}$ pode ser obtido a partir de cálculo direto, segundo (CIEMAT, 2002), que se baseia em experimentos empíricos. Em que (GUSTAVO, 2003):

- Para vento de baixa variabilidade $(\sigma / v(h) \approx$ $0,1): \quad \mathrm{k}=1.05 \cdot \sqrt{v(h)}$ (5)
- Para vento de baixa variabilidade $(\sigma / v(h) \approx$ $0,5): \quad \mathrm{k}=0.94 \cdot \sqrt{v(h)}$ (6)

- Para vento de baixa variabilidade $(\sigma / v(h) \approx$ $0,9): \quad \mathrm{k}=0.83 \cdot \sqrt{v(h)}$ (7)

onde $\sigma$ é o desvio padrão.

O valor de c pode ser obtido diretamente pela equação:

$$
c=\frac{v(h)}{\Gamma\left(1+\frac{1}{k}\right)}
$$

\subsection{Geração Eólica}

O principal foco da simulação do potencial eólico reside na necessidade de se estimar qual a potência média suprida pelas turbinas eólicas durante um mês normal de operação. Desta maneira, a seguir indicamos a forma pela qual encontramos o potencial.

A potência convertida por uma turbina eólica pode ser expressa através da equação:

$$
P_{\text {Turbina }}=\frac{1}{2} \cdot \rho \cdot A \cdot v^{3} \cdot C_{p}(\lambda, \theta)
$$

em que v é a velocidade de vento, $A$ é a área varrida pelas pás em $\mathrm{m}^{2}, \rho$ é a densidade do ar em $\mathrm{kg} / \mathrm{m}^{3}, \mathrm{Cp}(\lambda, \theta)$ é o coeficiente de potência, um parâmetro que pode ser calculado ou estimado para uma turbina eólica com base na eficiência da conversão eólica e eletromecânica, finalmente, $\mathrm{P}_{\text {turbina }}$ é a potência elétrica instantânea fornecida por uma turbina eólica em watts.

A Eq. (9) revela que a potência disponível é fortemente dependente da velocidade do vento: quando esta duplica, a potência aumenta oito vezes, mas duplicando a área varrida pelas pás da turbina, o aumento é só de duas vezes. Por outro lado, se a velocidade do vento desce para metade, a potência reduz-se a $12,5 \%$. Tudo isto explica a importância crítica da colocação das turbinas em locais com velocidades do vento elevadas no sucesso econômico dos projetos de energia eólica.

Quanto mais baixa a temperatura, mais moléculas de ar existem por metro cúbico. Por isso, o potencial eólico é maior quando a densidade do ar é maior, ou seja, quando está mais frio.

A densidade do ar $\rho$ varia de acordo com a altitude (pois tem uma relação com a pressão atmosférica) e com a temperatura do ar, deste modo a potência convertida por uma turbina eólica depende linearmente com a densidade 
do ar. A densidade do se relaciona com a pressão e com a temperatura conforme a equação seguinte:

$$
\rho=1.2929 \cdot \frac{P_{\text {Local }}}{760} \cdot \frac{273}{T_{\text {Local }}}
$$

em que $\mathrm{P}_{\text {Local }}$ é a pressão atmosférica em milímetros de mercúrio no local de instalação e $\mathrm{T}_{\text {Local }}$ é a temperatura local em Kelvin.

Verifica-se também que a potência convertida por uma turbina eólica também depende da velocidade do vento, neste caso com uma relação cúbica. Desta maneira a simples aplicação da velocidade média do vento nas equações conduz a um valor de potência média produzida pela turbina eólica muito inferior ao real.

Considerando que a potência de uma a turbina eólica também pode ser expressa pela Curva de Potência CP da mesma a equação da potência gerada por uma turbina eólica se torna mais simples. A curva CP já integra todas as funções apresentadas acima sendo ela medida em turbinas eólicas em condições de testes padrão o que demanda um fator de correção conforme indicado na equação a seguir:

$$
P_{\text {Turbina }}=f d \cdot C P(v)
$$

em que fd é um fator de correção para levar em consideração as variações da densidade do ar para diferentes alturas e temperaturas uma vez que as curvas de potência são calculadas para condições padrões, i.e. ao nível do mar e $15^{\circ} \mathrm{C}$ e $\mathrm{CP}(\mathrm{v})$ é a curva de potência da turbina eólica medida em condições padrões.

A potência elétrica média gerada por uma turbina eólica pode ser calculada através da seguinte equação:

$$
P_{\text {média }}=\int P_{\text {Turbina }}(v) \cdot f(v) d v
$$

em que $\mathrm{P}_{\text {Turbina }}(\mathrm{v})$ é a curva de potência da turbina eólica corrigida para o local como função do vento, $\mathrm{f}(\mathrm{v})$ é a função de densidade de probabilidade de vento neste caso de Weibull. Considerando a curva de potência de uma turbina eólica como sendo em pu (normalizada em relação a sua potência nominal), a potência média pode ser indicada como um valor em pu diretamente através da integração da Eq. (12).

$\mathrm{O}$ atrito entre a superfície terrestre e o vento tem como consequência um retardamento deste último. As camadas mais baixas de ar retardam as que lhe estão por cima, resultando numa variação da velocidade média do vento com a altura ao solo. O efeito da força de atrito vai-se desvanecendo até praticamente se anular a uma altura de aproximadamente 2.000 metros.
No solo, a condição fronteira obriga a que a velocidade do escoamento seja nula. A esta zona da atmosfera caracterizada pela variação da velocidade do vento com a altura, chama-se camada limite atmosférica; acima desta zona diz-se que a atmosfera é livre.

A figura 1 mostra a representação da curva do aerogerador da ENERCON E-92 2.3 MW e a tabela 1 mostra a curva de potência do aerogerador.

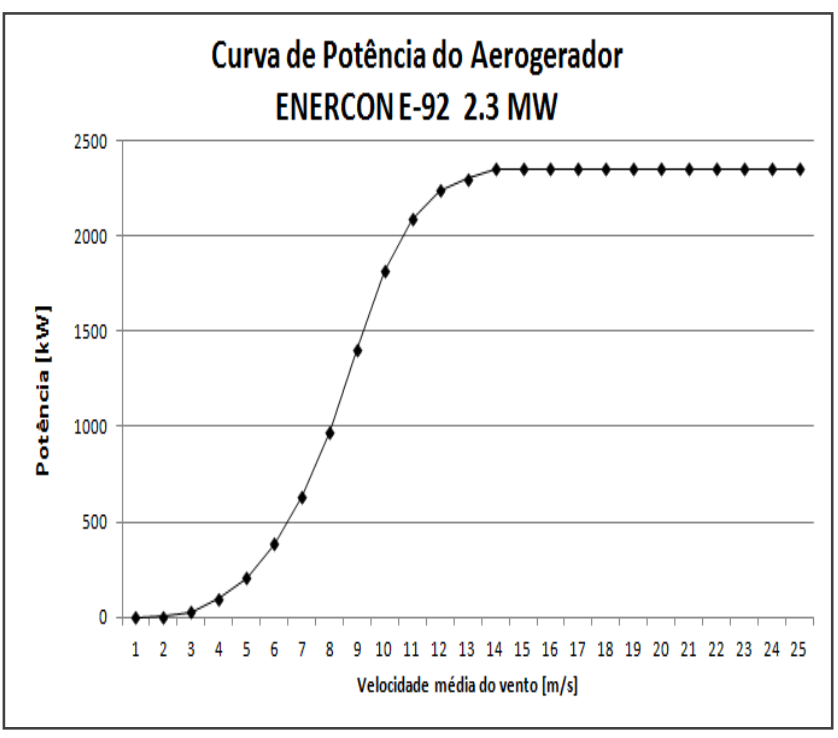

Figura 1 - Curva de Potência do Aerogerador.

Tabela 1 - Curva de Potência do Aerogerador ENERCON E-92 2.3 MW

\begin{tabular}{cc}
\hline $\mathbf{v}(\mathbf{m} / \mathbf{s})$ & Potência $(\mathbf{k W})$ \\
\hline 1,00 & 0 \\
2,00 & 3,6 \\
3,00 & 29,9 \\
4,00 & 98,2 \\
5,00 & 208,3 \\
6,00 & 384,3 \\
7,00 & 637,0 \\
8,00 & 975,8 \\
9,00 & 1403,6 \\
10,00 & 1817,8 \\
11,00 & 2088,7 \\
12,00 & 2237 \\
13,00 & 2300 \\
14,00 & 2350 \\
15,00 & 2350 \\
16,00 & 2350 \\
\hline
\end{tabular}




\begin{tabular}{ll}
\hline & \\
\hline 17,00 & 2350 \\
18,00 & 2350 \\
19,00 & 2350 \\
20,00 & 2350 \\
21,00 & 2350 \\
22,00 & 2350 \\
23,00 & 2350 \\
24,00 & 2350 \\
25,00 & 2350 \\
\hline
\end{tabular}

FONTE: ENERCON, Energy for the World,2011.

A energia anual esperada para cada aerogerador é determinada através da sequência de cálculos apresentada a seguir:

1. Utilizaram-se as velocidades médias mensais do período de janeiro de 2005 a dezembro de 2010 medidos à 10 metros de altura de 9 (nove) estações anemométricas em municípios do Estado de Pernambuco na região Nordeste do Brasil, essas velocidades são modeladas pela eq. (4) para as alturas de 85, 98 e 120 metros, para as torres eólicas hipotéticas;

2. A partir destes valores modelados é calculado o parâmetro k mensal eq. (5), (6) e (7) a escolha da equação adequada entre as três para o cálculo de $\mathrm{k}$ dependeu da variabilidade da velocidade do vento obtida pela equação $\sigma / v(h)$, onde $\sigma$ é o desvio padrão das velocidades médias mensais e $v(h)$ é a velocidade modelada por eq. (4), devendo-se respeitar as condições citadas anteriormente no presente trabalho. $\mathrm{O}$ parâmetro $\mathrm{c}$ mensal é calculado pela eq. (8);

3. A frequência calculada pela distribuição de Weibull é obtida por eq.(1) para os valores de k, c e $\mathrm{v}$, onde $v$ é a velocidade fornecida pelo fabricante do aerogerador a ser testada;

4. O valor da energia esperada (Ee) é calculado pela equação:

$E e=f(v) \cdot n \cdot P_{\text {Turbina }} \cdot \frac{m_{\text {ar local }}}{m_{\text {ar padrão }}}$

Onde $f(v)$ é a frequência da distribuição de Weibull, $n$ é número de horas, $P_{\text {Turbina }}$ é a potência $[\mathrm{kW}]$ do vento "aproveitada" pelo aerogerador (valores fornecidos pelo fabricante - Tabela 1), $m_{\text {ar local }}$ é massa específica do ar local $1 \mathrm{em}$ $\mathrm{kg} / \mathrm{m}^{3}$ e $m_{\text {ar padrão é a massa específica do ar }}$ padrão $\mathrm{em} \mathrm{kg} / \mathrm{m}^{3}$. Pode-se usar a função do MS Excel $2007 \quad$ =WEIBULL(x,alfa,beta,FALSE)*n
* $P_{\text {Turbina }} * m_{\text {ar local }} / m_{\text {ar padrão, onde } \mathbf{x} \text { é a }}$ velocidade $v$, alfa é $\mathrm{k}$ e beta $\mathrm{c}$;

5. O somatório dos valores representa a energia total esperada Eea(Total) $\mathrm{em} \mathrm{kWh}$ durante o ano.

\section{Estudo de Caso}

A área do Nordeste brasileiro é de aproximadamente $1.558 .196 \mathrm{~km}^{2}$, equivalente a $18 \%$ do território nacional e é a região que possui a maior costa litorânea. Está situado entre os paralelos de $01^{\circ} 02^{\prime} 30^{\prime \prime}$ de latitude norte e $18^{\circ} 20^{\prime}$ $07^{\prime \prime}$ de latitude sul e entre os meridianos de $34^{\circ} 47^{\prime} 30^{\prime \prime}$ e $48^{\circ} 45^{\prime} 24^{\prime \prime}$ a oeste do meridiano de Greenwich. Limita-se a norte e a leste com o Oceano Atlântico; ao sul com os estados de Minas Gerais e Espírito Santo e a oeste com os estados do Pará, Tocantins e Goiás.

O Nordeste possui importantes bacias hidrográficas, dentre as quais podemos destacar a Bacia do São Francisco. É a principal da região, formada pelos rios São Francisco e seus afluentes. São praticadas atividades de pesca, navegação e produção de energia elétrica pelas hidrelétricas de Três Marias, Sobradinho, Itaparica, Paulo Afonso, Apolonio Sales e Xingó, delimita as divisas naturais de Bahia com Pernambuco e também de Sergipe e Alagoas, que é onde está localizada sua foz.

O Estado de Pernambuco é uma das unidades da Federação brasileira localizada na região Nordeste, ocupa uma área de $98.146 \mathrm{~km} 2$. Esse espaço geográfico abrange diversas paisagens, constituídas a partir da interação entre os elementos naturais, sendo que os principais são: relevo, clima, vegetação e hidrografia.

O relevo do estado é formado basicamente por três tipos: planície costeira, planalto e depressão. Grande parte do território estadual, cerca de $76 \%$, possui um relevo relativamente plano, não ultrapassa os 600 metros de altitude. As planícies se encontram em áreas próximas ao litoral. À medida que se afasta do litoral a altitude aumenta, pode atingir até 1.200 metros.

Em Pernambuco são identificadas duas características climáticas: o clima tropical e o semiárido. O clima tropical ocorre nas áreas litorâneas denominadas de zona da mata. A temperatura é elevada, a média anual é de $24^{\circ} \mathrm{C}$. Quanto aos índices pluviométricos, a média anual é de $1.500 \mathrm{~mm}$. Nas áreas que predominam o clima semiárido as temperaturas são elevadas em boa parte do ano (cerca de $26^{\circ}$ ) e a quantidade de chuva é reduzida, algo em torno de $600 \mathrm{~mm}$ ao ano.

A hidrografia pernambucana é formada por vários rios, mas, sem dúvida, o principal é o São Francisco, uma vez 
que o mesmo é fundamental para a irrigação e demais atividades do sertanejo nordestino. Existem ainda os rios: Capibaribe, Ipojuca, Una, Pajeú e Jaboatão.

O regime de ventos da região Nordeste do Brasil está relacionado ao movimento latitudinal do Centro de Alta Pressão do Atlântico Sul. Segundo LIRA (1987), o sistema de circulação costeira no litoral de Pernambuco é regulado pela direção e pela intensidade dos ventos.

Quanto à sazonalidade dos ventos, todo o Nordeste apresenta ventos máximos no segundo semestre (inverno e primavera).

Utilizaram-se as vazões afluentes médias mensais da série histórica de 74 anos (1931 a 2010) do posto hidrológico da usina hidrelétrica de Sobradinho, em ONS (2011). Verifica-se uma das grandes dificuldades nas séries de vazões, que é a presença da sazonalidade devido aos períodos de cheia e seca do ano, isto acontece devido a grande variabilidade existente no período úmido, correspondente de novembro a abril, conforme Figura 2 e 3.

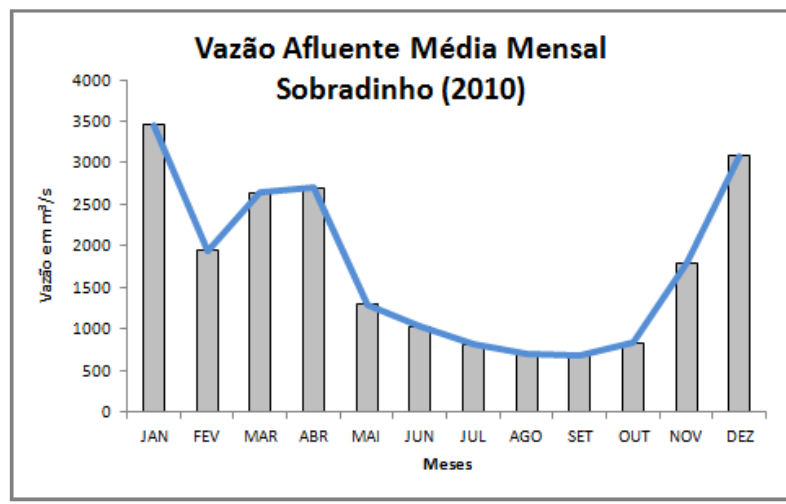

Figura 2 - Vazão Afluente de Sobradinho em m³/s, 2010, período seco.

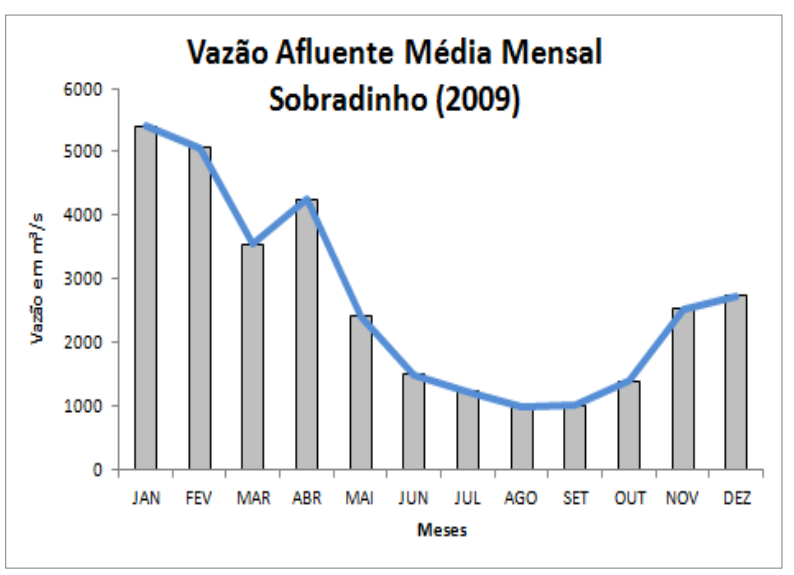

Figura 3 - Vazão Afluente de Sobradinho m³/s, 2009, período de cheia.
Utilizaram-se as velocidades médias mensais do período de janeiro de 2005 a dezembro de 2010 medidos à 10 metros de altura de 9 (nove) estações anemométricas em municípios do Estado de Pernambuco na região Nordeste do Brasil, juntamente com a curva de potência do aerogerador da ENERCON E-92 2.3 MW, conforme a Figura 1. As torres foram hipoteticamente instaladas com 85,98 e 120 metros de altura, que serviu de base para a concepção das fazendas eólicas hipotética, num arranjo com 456 aerogeradores instalados nas estações anemométricas potência instalada de 1050 MW equivalente à potência instalada na Usina Hidrelétrica de Sobradinho.

A Tabela 2 e 3 mostram a velocidade média mensal em $\mathrm{m} / \mathrm{s}$ a 85,98 e 120 metros de altura e altitude, de cada estação anemométrica, no ano de 2009 e 2010:

\begin{tabular}{|c|c|c|c|c|}
\hline Estação & $\mathrm{V}_{85}$ & $\mathrm{~V}_{98}$ & $\mathrm{~V}_{120}$ & $\begin{array}{c}\text { Altitude } \\
(\mathrm{m})\end{array}$ \\
\hline Arcoverde & 6,35 & 6,63 & 7,04 & 680,70 \\
\hline Cabrobó & 7,13 & 7,74 & 7,90 & 341,46 \\
\hline Garanhuns & 5,95 & 6,21 & 6,60 & 822,76 \\
\hline Ouricuri & 5,48 & 5,72 & 6,08 & 459,28 \\
\hline Pesqueira & 5,24 & 5,47 & 5,81 & 639,00 \\
\hline Petrolina & 5,12 & 5,34 & 5,67 & 370,46 \\
\hline Recife & 3,82 & 3,98 & 4,23 & 10,00 \\
\hline Surubim & 6,52 & 6,81 & 7,24 & 418,32 \\
\hline Triunfo & 7,95 & 8,30 & 8,82 & 1105,00 \\
\hline
\end{tabular}

Tabela 2 - Velocidade Média Mensal para 85, 98 e 120 metros de altura.

\begin{tabular}{|c|c|c|c|c|}
\hline Estação & $\mathrm{V}_{85}$ & $\mathrm{~V}_{98}$ & $\mathrm{~V}_{120}$ & $\begin{array}{c}\text { Altitude } \\
(\mathrm{m})\end{array}$ \\
\hline Arcoverde & 6,48 & 6,76 & 7,04 & 680,70 \\
\hline Cabrobó & 6,78 & 7,07 & 7,52 & 341,46 \\
\hline Garanhuns & 5,75 & 6,00 & 6,37 & 822,76 \\
\hline Ouricuri & 5,50 & 5,73 & 6,09 & 459,28 \\
\hline Pesqueira & 4,48 & 5,09 & 5,41 & 639,00 \\
\hline Petrolina & 5,51 & 5,75 & 6,11 & 370,46 \\
\hline Recife & 3,97 & 4,15 & 4,41 & 10,00 \\
\hline Surubim & 6,76 & 7,06 & 7,50 & 418,32 \\
\hline Triunfo & 8,68 & 9,06 & 9,62 & 1105,00 \\
\hline
\end{tabular}

Tabela 3 - Velocidade Média Mensal para 85, 98 e 120 metros de altura.

Os valores dos parâmetros $\mathrm{k}$ e c da distribuição de Weibull foram obtidos mensalmente da Eq. (5), (6) e (7) (satisfeitas às condições citadas sobre variabilidade que em foi em média menor que 0,1) e Eq. (8), respectivamente, e para a simulação da produtividade eólica anual se converteu através da Eq. (9) a velocidade média mensal do vento com coeficiente de rugosidade de 0,30 (Pequenas 
cidades com poucas árvores e arbustos) para as nove localidades na altura de 85,98 e 120 metros de altura, massa específica do ar local de $1,078 \mathrm{~kg} / \mathrm{m}^{3}$ e massa específica do ar padrão de $1,235 \mathrm{~kg} / \mathrm{m}^{3}$, a energia eólica.

A Figura 3, 4 e 5 mostram a curva da média mensal da velocidade do vento de todas as estações anemométrica para o ano de 2009, a 85, 98 e 120 metros de altura.

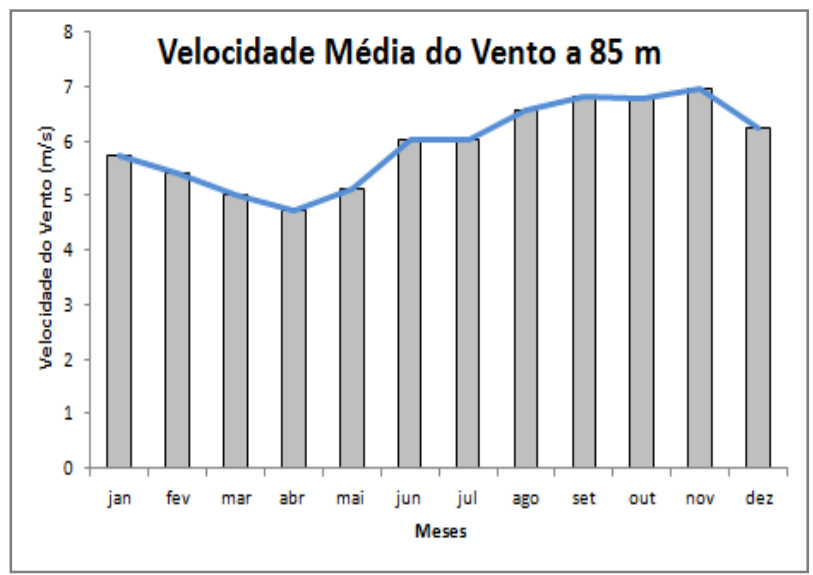

Figura 3 - Média Mensal da Velocidade do Vento das Estações Estudadas.

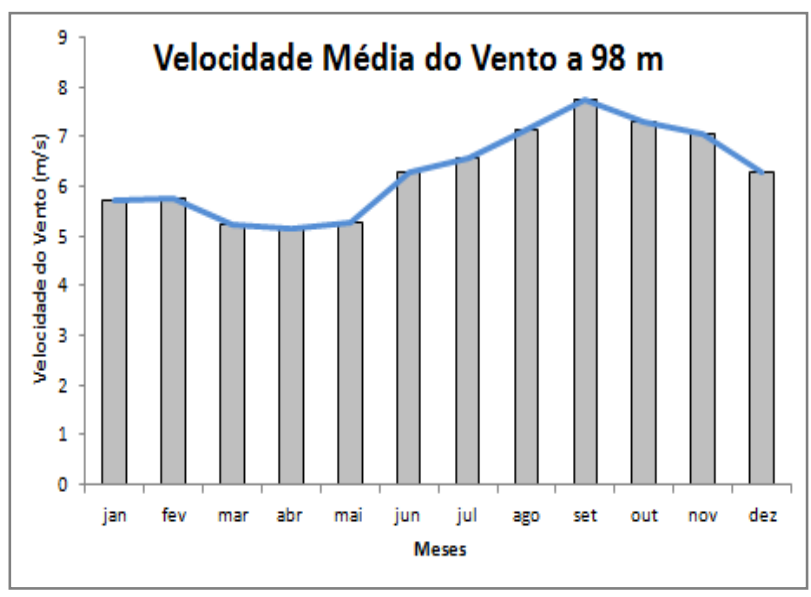

Figura 4 - Média Mensal da Velocidade do Vento das Estações Estudadas.

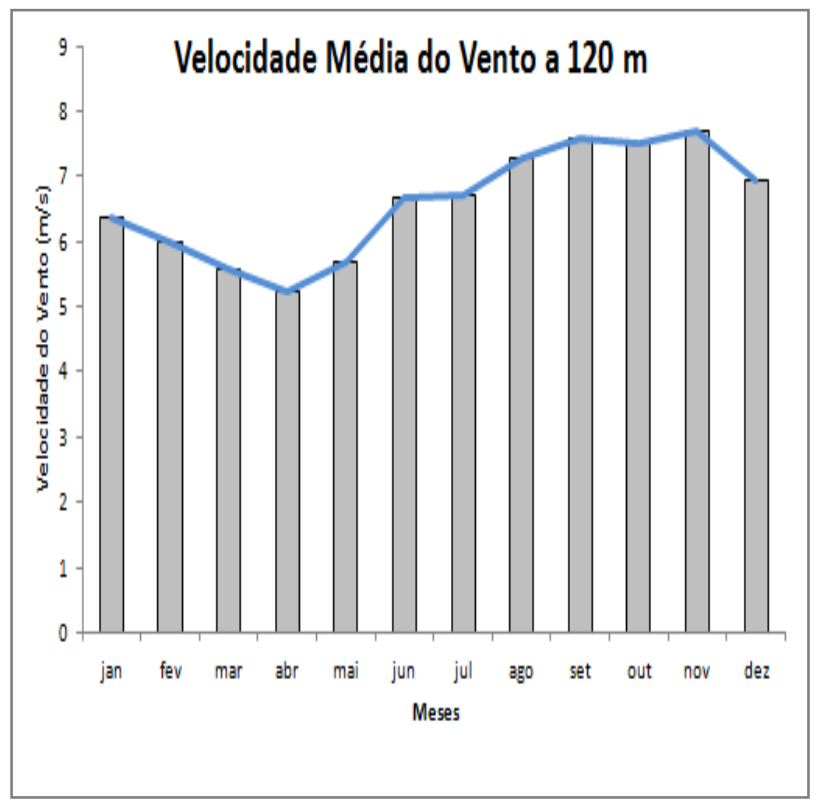

Figura 4 - Média Mensal da Velocidade do Vento das Estações Estudadas.

A Figura 6, 7 e 8 mostram a curva da média mensal da velocidade do vento de todas as estações anemométrica para o ano de 2010, a 85, 98 e 120 metros de altura.

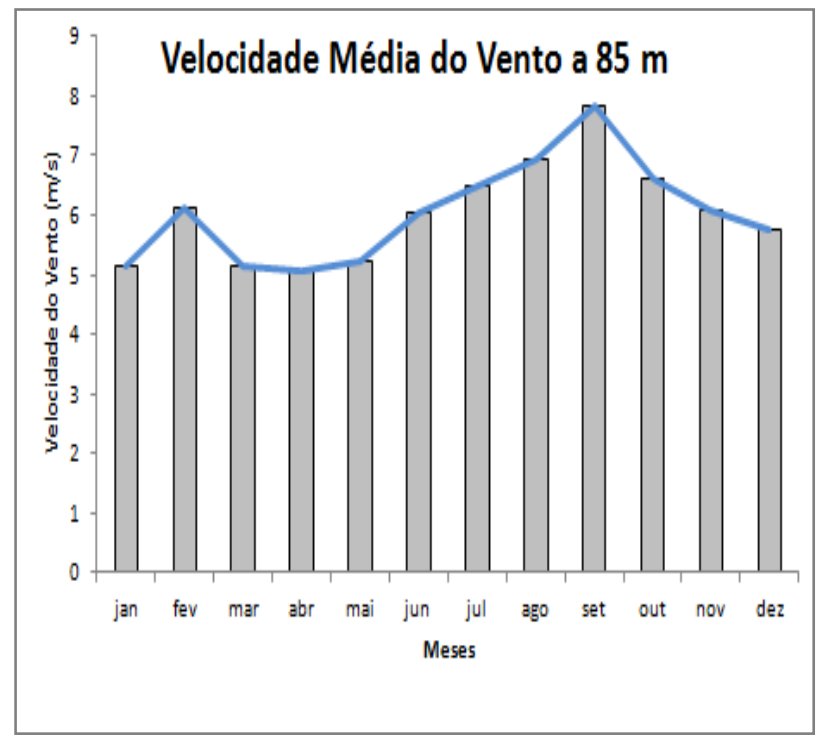

Figura 6 - Média Mensal da Velocidade do Vento das Estações Estudadas. 


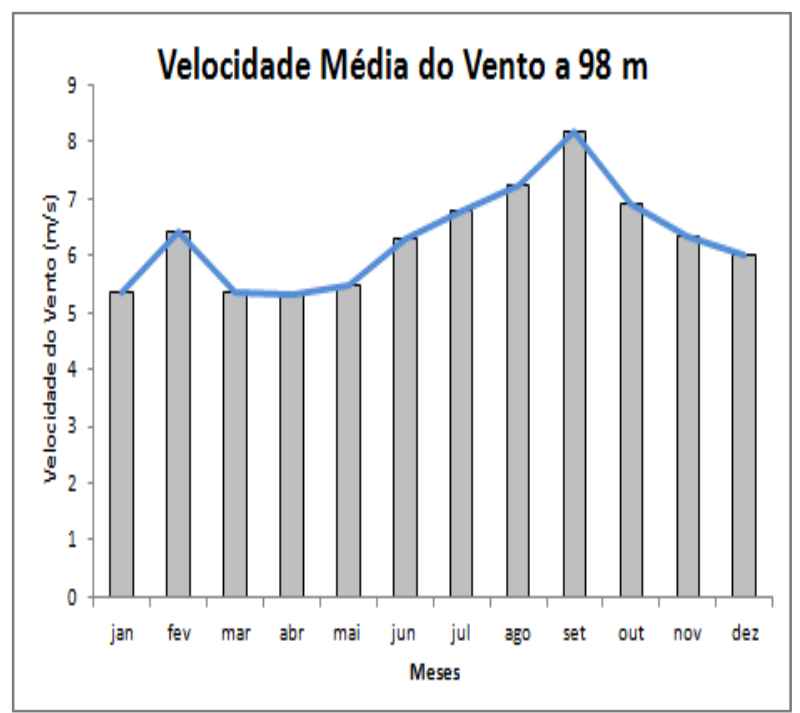

Figura 7 - Média Mensal da Velocidade do Vento das Estações Estudadas.

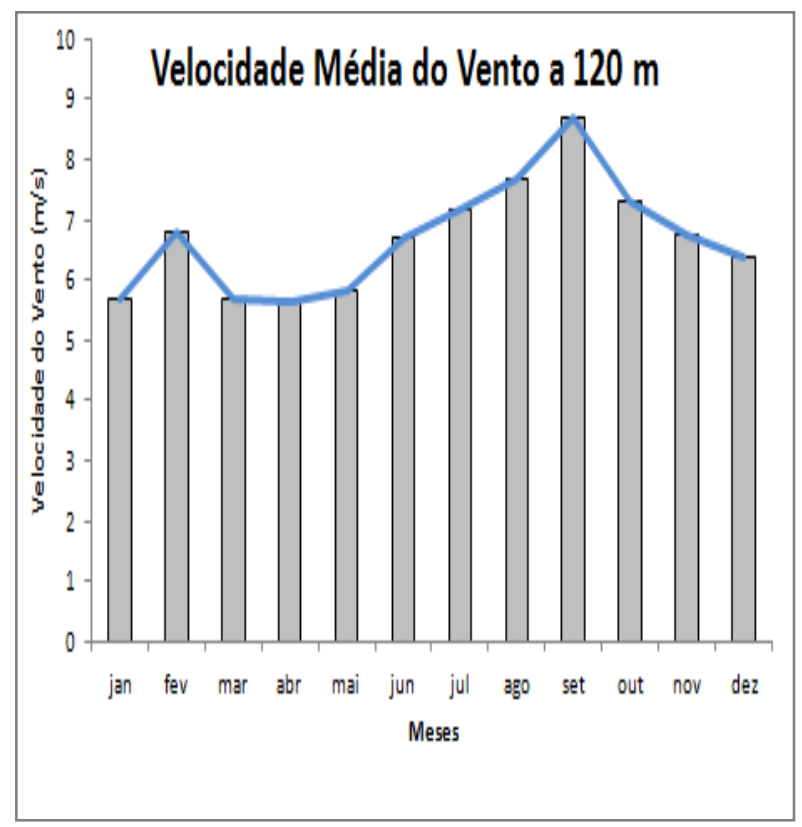

Figura 8 - Média Mensal da Velocidade do Vento das Estações Estudadas.

\section{Resultados e Discussão}

As Tabelas a seguir mostram os resultados obtidos pelos cálculos energéticos para cada altura no ano de 2009, em um período de cheia, ver vazão afluente Figura 2.

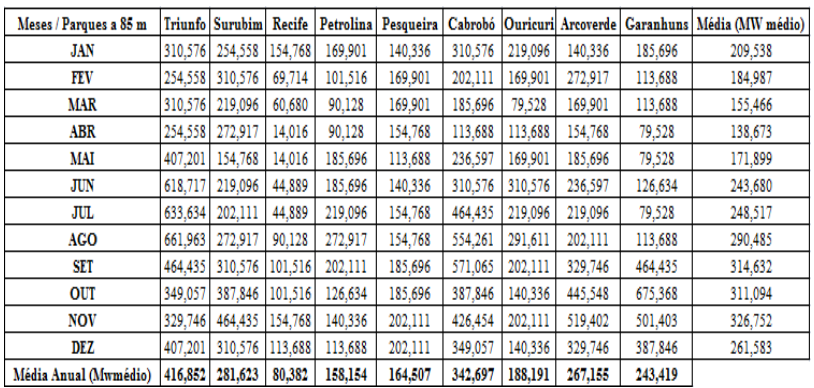

Tabela 4 - Cálculos Energéticos para Parques que Torres de 85 metros de Altura, no ano de 2009, período de cheia.

\begin{tabular}{|c|c|c|c|c|c|c|c|c|c|c|}
\hline Mleses / Parques a $98 \mathrm{~m}$ & Triunfo & Surubim & Recile & Petrolina & Pesqueira & Cabrobó & \begin{tabular}{|l|} 
Ouricuri \\
\end{tabular} & Arcorerde & Garanhuns & Média \\
\hline JAN & 340,746 & 281,04 & 172,661 & 199,278 & 156,758 & 340,746 & 242,874 & 156,738 & 206,538 & 231,941 \\
\hline IEV & 281,094 & 340,746 & 78,222 & 113,729 & 189,278 & 224,444 & 189,278 & 300,743 & 127,250 & 204,978 \\
\hline MAR & 340,746 & 242,874 & 68,112 & 101,041 & 189,278 & 206,558 & 89,207 & 189,278 & 127,250 & 172,706 \\
\hline$A B R$ & 281,094 & 300,743 & 15,676 & $101,04 !$ & 172,661 & 127,260 & $1.27,260$ & 172,661 & 89,207 & 154,178 \\
\hline IEI & 441,524 & 172,661 & 15,676 & 206,598 & 127,260 & 261,781 & 189,278 & 206,598 & 99,207 & 190,006 \\
\hline$\pi \mathrm{N}$ & 652,443 & 242,874 & 50,395 & 206,558 & 156,758 & 340,746 & 340,46 & 261,781 & 141,612 & 265,990 \\
\hline$\pi \mathrm{L}$ & 666,796 & 224,44 & 30,395 & 242,874 & 172,661 & 499934 & 242,874 & 242,874 & 99,207 & 270,228 \\
\hline $\mathrm{AGO}$ & 693,816 & 300,743 & 101,041 & 300,743 & 172,661 & 589,630 & 320,651 & 224,44 & 127,250 & $314,5.54$ \\
\hline SET & 4999934 & 340,746 & 113,729 & 224,44 & 206,558 & 606,134 & 224,444 & 360,954 & 499,934 & 341,875 \\
\hline OUT & 381,204 & 421,553 & 113,729 & 141,612 & 206,558 & 421.553 & $1.56,758$ & 480,766 & 706,509 & 336,693 \\
\hline NOV & 360994 & 499,934 & 172,661 & 156,758 & 224,444 & 461,279 & 224,44 & 355,114 & 537,145 & 334,748 \\
\hline $\mathrm{DEZ}$ & $44,1,24$ & 340,746 & 127,260 & 127,260 & 22,444 & 381,204 & 156,58 & 360,954 & $421,5,53$ & 286,856 \\
\hline ull (NW médio) & 448,489 & 309,096 & 89,964 & 175,991 & 183,276 & 371,772 & 208,714 & 292,740 & 263,5359 & \\
\hline
\end{tabular}

Tabela 5 - Cálculos Energéticos para Parques que Torres de 98 metros de Altura, no ano de 2009, período de cheia.

\begin{tabular}{|c|c|c|c|c|c|c|c|c|c|c|}
\hline Neses $/$ Paryes a 120 m & Iriunfo & Survbim & Recile & Petrolina & \begin{tabular}{|l|} 
Pesquetin \\
\end{tabular} & Cabrobio & Ouricuri & \begin{tabular}{|l|} 
Arcoverde \\
\end{tabular} & Garnhuns & Media \\
\hline J.AN & 386,367 & 321,966 & 201,156 & 219990 & 183,027 & 386,367 & 279,962 & 183,02 & 239,56 & 266,816 \\
\hline FV & 321996 & 386,367 & 92,118 & 133,485 & 219990 & 259,474 & 219,990 & $343,3,64$ & 149,139 & 266,214 \\
\hline WAR & 386,367 & 279,962 & 80,253 & 118,47 & 219,990 & 239,56 & 100952 & 219990 & 149,139 & 199,873 \\
\hline$A B R$ & 321996 & $34,3,34$ & 188402 & 118,47 & 201,156 & 149,139 & 149,139 & 201,156 & 109952 & 178,62 \\
\hline ILII & 491,701 & 201,156 & 18,402 & 299,566 & 149,139 & 300,832 & 219,990 & 239,56 & 104952 & 218,343 \\
\hline$\pi \mathrm{x}$ & 6979937 & 279,92 & 59,415 & 239,456 & 183,07 & 3366,367 & 386,367 & 30,832 & 166,669 & 299,92 \\
\hline$\pi$ & 711,222 & 259,774 & 59,415 & 279,962 & 201,156 & 350,775 & 279,92 & 279,962 & 10,952 & 302,987 \\
\hline$A G O$ & 733998 & $34,3,34$ & 118,77 & 34,364 & 201,156 & 638,601 & 364,50 & 259,74 & 149,139 & 30,519 \\
\hline SEI & 350,775 & 386,367 & 133,455 & 259,47 & 239,456 & 654,369 & 259,774 & 407,344 & 550,715 & 322,45 \\
\hline $0 \mathrm{OT}$ & 429,171 & 471,169 & 133,455 & 165,669 & 239,456 & 47,169 & 183,027 & 531,50 & 77,72 & $37+655$ \\
\hline SOT & 407,834 & 550,75 & 201,136 & 183,027 & 259,774 & 511,84 & 259,774 & 6052,25 & 587,640 & 366,272 \\
\hline DEZ & 491,01 & 386,367 & 199,139 & $19,1,139$ & 259,774 & 49,171 & 188,027 & 407,344 & 47,169 & 365224 \\
\hline MEdedia, Anul (NW mádio) & 494,420 & 350,860 & 105,431 & 204,210 & 213,042 & 414,797 & 240,851 & 331,642 & 293,704 & \\
\hline
\end{tabular}

Tabela 6 - Cálculos Energéticos para Parques que Torres de 120 metros de Altura, no ano de 2009, período de cheia.

As Tabelas a seguir mostram os resultados obtidos pelos cálculos energéticos para cada altura no ano de 2010, em um período de seca, ver vazão afluente Figura 1.

\begin{tabular}{|c|c|c|c|c|c|c|c|c|c|c|}
\hline Neses / Parques 85 m & Iriunfo & Surntim & Reilif & Petroling & Pesqueira & Cabroto & Ouricuri & Arconerde & Garahums & Media (NIT médio) \\
\hline JAY & 272917 & 169901 & 524,40 & 140,336 & 154,768 & 254558 & 79.528 & 236597 & 113,688 & 163,566 \\
\hline $\mathrm{FV}$ & 368,44 & 368,44 & 79,228 & 154,768 & 140,336 & 483,068 & 1699,901 & 140,336 & $368, \mathrm{HH}$ & 252385 \\
\hline MHR & 272917 & 254558 & 60,680 & 100,336 & 100,336 & 185,696 & 90,128 & 140,336 & 169,01 & 161,654 \\
\hline$A B R$ & 45,448 & 202,111 & 52410 & 169,901 & 101,516 & 113,688 & 113,688 & 101516 & 154,768 & 161,683 \\
\hline MAI & 45,48 & 24,558 & 38,094 & 169,901 & 113,688 & 113,688 & 113,688 & 2545538 & 101,516 & 178,360 \\
\hline$\pi x$ & 6882,267 & 266,597 & 4,889 & 202,111 & 101516 & 236,597 & 236,597 & 399,057 & 113,688 & 25,400 \\
\hline$\pi$ & 700,662 & 2365997 & 60,680 & 236,597 & 126,634 & 349,057 & 349,057 & 291,611 & 185,666 & 281,84 \\
\hline$A G O$ & 764,799 & 368,44 & 79,228 & 291,611 & 126,634 & $368 \mathrm{H4}$ & 368,44 & 399,657 & 154,768 & 319,001 \\
\hline SEI & 734.875 & 407201 & 154,768 & 310,576 & 154,768 & 734.875 & 387,86 & 407201 & 291,611 & 398,191 \\
\hline OUT & 501,403 & 38,446 & 126,634 & 185,696 & 169,901 & 501,03 & 185,696 & 329,74 & 254558 & 293,654 \\
\hline sor & 219,096 & 407,201 & 140.336 & 154,768 & 154,768 & 1699,01 & 1699901 & 455.48 & 329,76 & 213,474 \\
\hline DEZ & 272917 & 368,44 & 113,688 & 113,688 & 154,768 & 1699,01 & 90,128 & 39,746 & 329,76 & 215,992 \\
\hline Mediat. & 473,949 & 305,159 & 83,637 & 189,191 & 136,636 & 306,740 & 196,217 & 281,276 & 214,011 & \\
\hline
\end{tabular}


Tabela 7 - Cálculos Energéticos para Parques que Torres de 85 metros de Altura, no ano de 2010, período de seca.

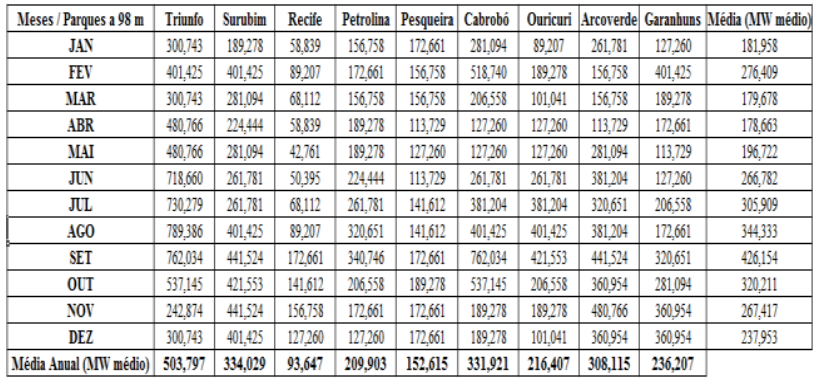

Tabela 8 - Cálculos Energéticos para Parques que Torres de 98 metros de Altura, no ano de 2010, período de seca.

\begin{tabular}{|c|c|c|c|c|c|c|c|c|c|c|}
\hline Meses / Parques a $120 \mathrm{~m}$ & Triunfo & Surubim & Recile & Petrolina & Pesquein & Cabrobó & Ouricuri & Arcoverde & Garanhuns & Média (MW médio) \\
\hline JAY & 343,364 & 219990 & 69,356 & 183,027 & 201,156 & 321,996 & 104,952 & 30,832 & 149,139 & 210,423 \\
\hline FEV & 450,305 & 450305 & $10,9,52$ & 201,156 & 183,027 & 569,482 & 219990 & 183,027 & 450305 & 312,305 \\
\hline IAAR & 343,364 & 321,996 & 80,253 & 183,027 & 183,027 & 239,456 & 118,747 & 183,027 & 219990 & 208,099 \\
\hline$A B R$ & 531,550 & 259,474 & 69,356 & 219990 & 133,485 & 149,139 & 149,139 & 133,485 & 201,156 & 205,197 \\
\hline IHI & 531,550 & 321.996 & 50,414 & 219990 & 149,139 & 149,139 & 149,139 & 321996 & 133,485 & 225,205 \\
\hline$\pi \mathrm{x}$ & 758,394 & 300,832 & 59,415 & 259,474 & 133,485 & 300,832 & 300,832 & 429,171 & 149,139 & 29,064 \\
\hline$\pi \mathrm{L}$ & 7687,78 & 300,822 & 80253 & 300,832 & 165,669 & 42,171 & 429,171 & 364,850 & 239,456 & 342110 \\
\hline$A G O$ & 820,053 & 450305 & 10,952 & 364,850 & 165,669 & 450,305 & 450,305 & 429,171 & 201,156 & 381,863 \\
\hline SET & 796,652 & 491,701 & 201,156 & 386,367 & 201,156 & 796,652 & 471,169 & 491,701 & 364,850 & 466,823 \\
\hline OUI & 587,640 & 471,169 & 165,669 & 239,456 & 219990 & 587,640 & 239,456 & 407,834 & 321996 & 360,044 \\
\hline $\mathrm{SON}$ & 279,962 & 491,701 & 183,027 & 201,156 & 201,156 & 219,990 & 219990 & 531,50 & 407,834 & 304,041 \\
\hline DEZ & 343,364 & 450305 & 149,139 & 199,139 & 201,156 & 219,990 & 118,747 & 407,834 & 407,834 & 271,945 \\
\hline I $\mathrm{M}$ & 546,246 & 377,550 & 109,828 & 242,372 & 178,176 & 369,483 & 247,636 & 348,706 & 270,528 & \\
\hline
\end{tabular}

Tabela 9 - Cálculos Energéticos para Parques que Torres de120 metros de Altura, no ano de 2010, período de seca.

A partir destes resultados da geração eólica dos parques hipotéticos, obtidos por métodos aqui citados, e de posse das vazões mensais naturais do posto hidrológico da Usina de Sobradinho, especificamente para o ano de 2009 (cheia) e 2010 (seca) que foram períodos em que há variação significativa no período de 2005 a 2012, ou seja, o período de 2009 houve a maior cheia e 2010 o período de maior seca, e juntamente baseado na informação de PORTO et al. (2002) a produtibilidade na cascata do São Francisco onde cada $100 \mathrm{MW}$ médios produzidos de fonte eólica proporcionaria uma economia de água da ordem de $40 \mathrm{~m}^{3} / \mathrm{s}$, foram obtidos os seguintes resultados, referentes a economia na vazão afluente média como mostram as Tabelas:

\begin{tabular}{|c|c|c|c|}
\hline \multicolumn{4}{|c|}{ ECONOMIA EM m³3/s PARA GERAÇÃO EÓlLCA A $85 \mathrm{~m}$ de ALTURA } \\
\hline Meses & VAZÃO $\left(\mathrm{m}^{3} / \mathrm{s}\right)$ Sobradinho & VAZÃo $\left(\mathrm{m}^{3} / \mathrm{s}\right)$ Economizada & Perc(\%) \\
\hline JAN & 5409 & 84 & 1,55 \\
\hline FEV & 5072 & 74 & 1,46 \\
\hline MAR & 3542 & 62 & 1,76 \\
\hline $\mathrm{ABR}$ & 4254 & 55 & 1,30 \\
\hline MAI & 2410 & 69 & 2,85 \\
\hline $\mathrm{JUN}$ & 1492 & 97 & 6,53 \\
\hline $\mathrm{JL}$ & 1236 & 99 & 8,04 \\
\hline $\mathrm{AG0}$ & 976 & 116 & 11,90 \\
\hline SET & 1019 & 126 & 12,35 \\
\hline OUT & 1393 & 124 & 8,93 \\
\hline $\mathrm{NOV}$ & 2534 & 131 & 5,16 \\
\hline \multirow[t]{2}{*}{$\mathrm{DEZ}$} & 2730 & 105 & 3,83 \\
\hline & Média $=2672$ & Soma $=1143$ & Média $=5,47$ \\
\hline
\end{tabular}

Tabela 10 - Vazão Economizada no Período de Cheia (2009).

\begin{tabular}{|c|c|c|c|}
\hline \multicolumn{4}{|c|}{ ECONOMIIA EM m³/s PARA GERAC̣̃̃0 EÓLICA A $98 \mathrm{~m}$ de ALTURA } \\
\hline Meses & VAZÃ0 $\left(\mathrm{m}^{3} / \mathrm{s}\right)$ Sobradinho & VAZÃ0 $\left(\mathrm{m}^{3} / \mathrm{s}\right)$ Economizada & $\operatorname{Perc}(\%)$ \\
\hline JAN & 5409 & 93 & 1,72 \\
\hline FEV & 5072 & 82 & 1,62 \\
\hline MAR & 3542 & 69 & 1,95 \\
\hline$A B R$ & 4254 & 62 & 1,45 \\
\hline MAI & 2410 & 76 & 3,15 \\
\hline $\mathrm{JU}$ & 1492 & 106 & 7,13 \\
\hline $\mathrm{JL}$ & 1236 & 108 & 8,74 \\
\hline AG0 & 976 & 126 & 12,89 \\
\hline SET & 1019 & 137 & 13,42 \\
\hline OUT & 1393 & 135 & 9,67 \\
\hline $\mathrm{NOV}$ & 2534 & 142 & 5,60 \\
\hline \multirow[t]{2}{*}{ DEZ } & 2730 & 115 & 4,20 \\
\hline & Média $=2672$ & Soma $=1250$ & Média $=\mathbf{5}, 96$ \\
\hline
\end{tabular}

Tabela 11 - Vazão Economizada no Período de Cheia (2009). 


\begin{tabular}{|l|c|c|c|}
\hline \multicolumn{5}{|c|}{ ECONOMIA EM m ${ }^{3} / \mathrm{s}$ PARA GERAÇ̃̃o EÓLICA A $120 \mathrm{~m}$ de ALTURA } \\
\hline Meses & VAZÃ0 $\left(\mathrm{m}^{3} / \mathrm{s}\right)$ Sobradinho & VAZÃ0 $\left(\mathrm{m}^{3} / \mathrm{s}\right)$ Economizada & Perc(\%) \\
\hline JAN & 5409 & 107 & 1,97 \\
\hline FEV & 5072 & 94 & 1,86 \\
\hline MAR & 3542 & 80 & 2,26 \\
\hline ABR & 4254 & 71 & 1,68 \\
\hline MAI & 2410 & 87 & 3,62 \\
\hline JUN & 1492 & 120 & 8,04 \\
\hline JUL & 1236 & 121 & 9,80 \\
\hline AG0 & 976 & 140 & 14,36 \\
\hline SET & 1019 & 153 & 15,01 \\
\hline OUT & 1393 & 150 & 10,76 \\
\hline NOV & 2534 & 159 & 6,25 \\
\hline DEZ & 2730 & 130 & 4,76 \\
\hline \multicolumn{2}{|c|}{ Média $=2672$} & Soma $=1413$ & Média $=6,70$ \\
\hline
\end{tabular}

Tabela 12 - Vazão Economizada no Período de Cheia (2009).

\begin{tabular}{|c|c|c|c|}
\hline \multicolumn{4}{|c|}{ ECONOMIA EM m³/s PARA GERAÇÃO EÓLICA A $85 \mathrm{~m}$ de ALTURA } \\
\hline Meses & VAZÃo $\left(\mathrm{m}^{3} / \mathrm{s}\right)$ Sobradinho & VAZÃ̃o $\left(\mathrm{m}^{3} / \mathrm{s}\right)$ Economizada & Perc(\%) \\
\hline $\mathrm{JAN}$ & 3459 & 66 & 1,89 \\
\hline FEV & 1939 & 101 & 5,21 \\
\hline MAR & 2642 & 65 & 2,45 \\
\hline$A B R$ & 2701 & 65 & 2,39 \\
\hline MAI & 1289 & 71 & 5,54 \\
\hline$\pi \mathrm{N}$ & 1025 & 98 & 9,58 \\
\hline$\pi \mathrm{L}$ & 815 & 113 & 13,83 \\
\hline AG0 & 690 & 128 & 18,49 \\
\hline SET & 683 & 159 & 23,33 \\
\hline OUT & 831 & 117 & 14,13 \\
\hline $\mathrm{NOV}$ & 1794 & 97 & 5,43 \\
\hline \multirow[t]{2}{*}{ DEZ } & 3086 & 86 & 2,80 \\
\hline & Média=1746 & Soma $=1166$ & Média $=8,76$ \\
\hline
\end{tabular}

Tabela 13 - Vazão Economizada no Período de Seca (2010).

\begin{tabular}{|c|c|c|c|}
\hline \multicolumn{4}{|c|}{ ECONONIA EM m³/s PARA GERAC̦̃̃o EÓLICA A $98 \mathrm{~m}$ de ALTURA } \\
\hline Meses & VAZÃ0 $\left(\mathrm{m}^{3} / \mathrm{s}\right)$ Sobradinho & VAZÃ0 $\left(\mathrm{m}^{3} / \mathrm{s}\right)$ Economizada & Perc(\%) \\
\hline JAN & 3459 & 73 & 2,10 \\
\hline FEV & 1939 & 111 & 5,70 \\
\hline MAR & 2642 & 72 & 2,72 \\
\hline ABR & 2701 & 71 & 2,65 \\
\hline MAI & 1289 & 79 & 6,11 \\
\hline $\mathrm{JN}$ & 1025 & 107 & 10,41 \\
\hline $\mathrm{JL}$ & 815 & 122 & 15,01 \\
\hline AGO & 690 & 138 & 19,96 \\
\hline SET & 683 & 170 & 24,97 \\
\hline OUT & 831 & 128 & 15,41 \\
\hline $\mathrm{NOV}$ & 1794 & 107 & 5,96 \\
\hline DEZ & 3086 & 95 & 3,08 \\
\hline & Média $=1746$ & Soma $=1273$ & Média $=9,51$ \\
\hline
\end{tabular}

Tabela 14 - Vazão Economizada no Período de Seca (2010).

\begin{tabular}{|c|c|c|c|}
\hline \multicolumn{4}{|c|}{ ECONOMII EM m ms PARA GERAÇÃ0 EÓLICA A $120 \mathrm{~m}$ de ALTURA } \\
\hline Meses & VAZÃ0 $\left(\mathrm{m}^{3} / \mathrm{s}\right)$ Sobradinho & VAZÃ0 $\left(\mathrm{m}^{3} / \mathrm{s}\right)$ Economizada & $\operatorname{Perc}(\%)$ \\
\hline JAN & 3459 & 84 & 2,43 \\
\hline $\mathrm{FEV}$ & 1939 & 125 & 6,45 \\
\hline MAR & 2642 & 83 & 3,15 \\
\hline $\mathrm{ABR}$ & 2701 & 82 & 3,04 \\
\hline MAI & 1289 & 90 & 6,99 \\
\hline $\mathrm{JU}$ & 1025 & 120 & 11,07 \\
\hline $\mathrm{JL}$ & 815 & 137 & 16,79 \\
\hline AG0 & 690 & 153 & 22,13 \\
\hline SET & 683 & 187 & 27,35 \\
\hline OUT & 831 & 144 & 17,33 \\
\hline NOV & 1794 & 122 & 6,78 \\
\hline $\mathrm{DEZ}$ & 3086 & 109 & 3,53 \\
\hline & Média $=1746$ & Soma $=1435$ & Média $=10,04$ \\
\hline
\end{tabular}

Tabela 15 - Vazão Economizada no Período de Seca (2010). 
Realizou-se um comparativo com dois períodos, cheia e seca, no intuito de demonstrar a eficiência da energia eólica nos dois períodos, comparando com a vazão afluente da Usina Hidrelétrica de Sobradinho. Comprovou-se, hipoteticamente, que a geração de energia eólica simulada nas três alturas $(85,98$ e 120$)$ tiveram praticamente os mesmos resultados, concluindo a significativa importância para o setor elétrico o estudo da complementaridade hidroeólica, mesmo em períodos inversos (seca e chuva).

Neste comparativo com um período de cheia e com um período de seca dos rios, mostrando que há uma variação significativa do período de cheia para um período de seca, na energia eólica gerada, ambos os comparativos são feitos com a vazão afluente da Usina Hidrelétrica de Sobradinho. Comprovou-se neste estudo hipotético que a variação de energia eólica gerada é mínima, pois a geração eólica no período de seca foi de aproximadamente 269 $\mathrm{MWh} / \mathrm{h}$ e no período de cheia foi de $264 \mathrm{MWh} / \mathrm{h}$. As simulações realizadas com a potência hipotética instalada de $1050 \mathrm{MW}$ alocadas em 9 (nove) municípios do Estado de Pernambuco na região Nordeste do Brasil, proporcionaram uma contribuição anual de produção eólica na ordem de 29,06 GWh/ano média no período seco e de 28,54 GWh/ano média no período de cheia, com 456 aerogeradores de $2.300 \mathrm{~kW}$ média distribuídos pelas fazendas eólicas a 85,98 e 120 metros de altura.

O fator de capacidade mede o desempenho de uma usina eólica no aspecto energético. Defini-se como a relação entre a potência média a potência máxima de uma usina. As usinas de base têm usualmente fatores de capacidade elevados, enquanto as de ponta apresentam fatores de capacidade mais baixos. Os valores obtidos para as simulações foram superiores ao valor típico esperado para as turbinas eólicas modernas $(0,30)$.

A utilização anual da potência instalada, em horas, é calculada pela divisão da energia produzida pela potência nominal. Os valores em ficaram em média de 1000 a 3600 horas.

Nota-se que a regularização da vazão do rio São Francisco pode receber uma grande colaboração de forma natural do aproveitamento eólico, principalmente no período seco que é o segundo semestre do ano, onde o maior potencial acontece por grande influência dos ventos alísios.

A Figura 9 e 10 apresentam uma comparação das vazões naturais afluentes no rio São Francisco com a média mensal da geração eólica de energia esperada nas estações anemométricas instaladas no Nordeste. Observa-se que o elevado potencial eólico da região Nordeste pode-se induzir que a contribuição da energia eólica a estabilização sazonal da oferta de energia elétrica pode advir de vários Estados da região, principalmente no segundo semestre do ano, período em que o potencial eólico na região é mais elevado e registram-se as menores vazões no rio São Francisco.

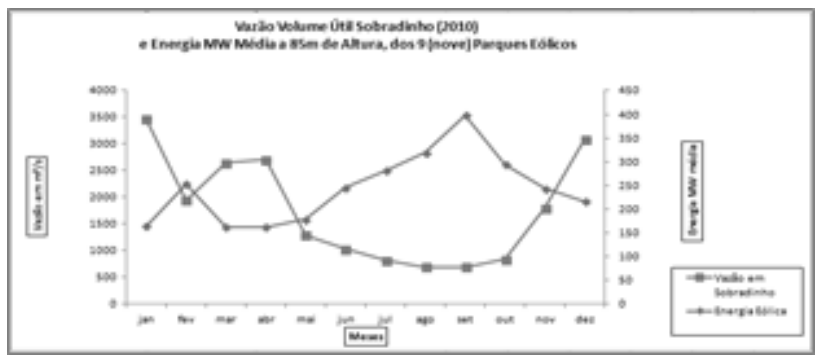

Figura 9 - Comparação das Vazões Afluentes de Sobradinho com a Média Mensal da Geração Eólica em um período de seca (2010)

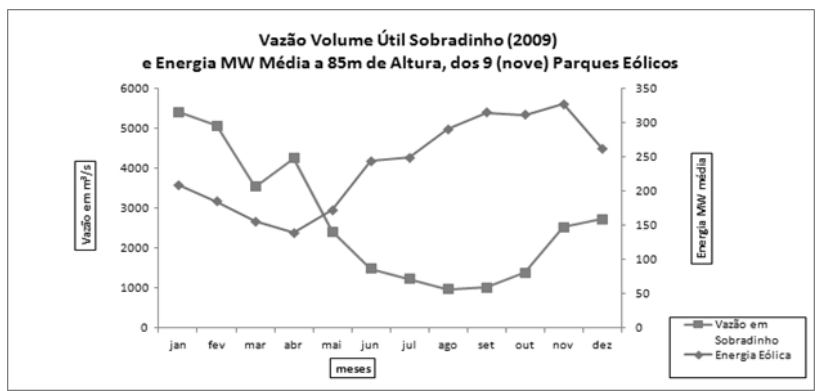

Figura 10 - Comparação das Vazões Afluentes de Sobradinho com a Média Mensal da Geração Eólica em um período de cheia (2009).

Observa-se na Figura 1 que o elevado potencial eólico da região Nordeste do Brasil pode-se induzir que a contribuição da energia eólica a estabilização sazonal da oferta de energia elétrica pode advir de vários municípios da região, principalmente no segundo semestre do ano, período em que o potencial eólico na região é mais elevado e registram-se as menores vazões no rio São Francisco.

A comprovação da existência de uma grande complementaridade sazonal entre os regimes hidráulicos das principais bacias e o regime eólico, como apresentado na Figura 9 em um período de seca, imputa significativos benefícios ao sistema interligado nacional, indicando ser possível adicionar maiores volumes de energia ao sistema exatamente nos períodos secos.

Observa-se também que na Figura 10, quando ocorre o período de cheia e baseado nos resultados obtidos pelos cálculos, percebe-se que há uma variação pequena e significativa entre períodos de seca e períodos de cheia, mostrando que o aproveitamento da energia eólica pode ser feito tanto em um período de seca como em um período de cheia, de forma benéfica, visto que o período de seca 
obteve ligeiramente melhor desempenho em todas as alturas simuladas, comparado à produtibilidade do período de cheia. Cabendo ao Governo criar as condições de desenvolvimento da política energética do país.

\section{Conclusão}

O estudo hipotético provou que a regularização da vazão do rio São Francisco, mais precisamente na barragem de Sobradinho, pode receber grandes quantidades de energia elétrica de forma eólica, evitando que se tenha que utilizar a água do rio São Francisco, inclusive no segundo semestre do ano, em que ocorrem as menores vazões afluentes e onde ocorre o maior potencial de energia por grande influência dos ventos alísios. Salientandose que a usina hidrelétrica de Sobradinho é sujeita à múltiplos usos e todo este estudo inicial teve referencia mensal, cabendo futuramente uma análise semanal ou diário para se verificar a real viabilidade operativa desse parque eólico.

Finalmente, as informações geradas servem como importante ferramenta no planejamento e nas tomadas de decisões de empresas, órgãos gestores e do próprio Governo. Entre as principais áreas beneficiadas estão: a agricultura, recursos hídricos, estudos científicos, geração de energia, defesa civil, transportes, saúde, turismo, lazer, etc.

\section{Referências}

[1] ONS - Operador Nacional do Sistema Elétrico, (2011). Relatório de Vazões Afluentes Médias Mensais nos Aproveitamentos Hidrelétricos, período de 1931 a 2010.

[2] CAMARGO, ARILDE SUTIL GABRIEL DE (2005) “Análise da operação das usinas eólicas de Camelinho e Palmas e avaliação do potencial eólico de localidades no Paraná”. Dissertação (Mestrado em Tecnologia) . Curitiba: CEFETPR,.

[3] SILVA, NEILTON FIDELIS (2006 ) "Fontes de Energia Renováveis Complementares na Expansão do Setor Elétrico Brasileiro: O Caso da Energia Eólica “ [Rio de Janeiro] 2006 VIII, 263 p. 29,7 cm (COPPE/UFRJ, D.Sc., Planejamento Energético.

[4] BERNARDO B. DA SILVA, JAKSON J. A. ALVES, ENILSON P. CAVALCANTI \& RENILSON T. DANTAS "Potencial eólico na direção predominante do vento no Nordeste brasileiro", Universidade Federal de Campina Grande, 2002.
[5] MARINHO, MANOEL HENRIQUE DA NÓBREGA "OFERTA DE ENERGIA ATRAVÉS DA COMPLEMENTARIDADE SAZONAL HIDRO-EÓLICA NO ESTADO DE PERNAMBUCO”, Universidade de Pernambuco.

[6] MARINHO, M. H. N (2010) "Previsão de Afluências Utilizando Redes Neurais para Complementaridade Hidro-Eólica Visando a Oferta de Energia Elétrica", Departamento de Engenharia Elétrica e Sistemas de Potência (DEESP) da Universidade Federal de Pernambuco (UFPE).

[7] SALLES, ANA CLAUDIA NIOAC DE (2004) “ Metodologias de Análise de Risco para Avaliação Financeira de Projetos de Geração Eólica" ,Tese de Mestrado. [Rio de Janeiro] 2004 X, 83 p. 29,7 cm (COPPE/UFRJ, M.Sc., Planejamento Energético, 2004).

[8] SILVA, GUSTAVO RODRIGUES (2003) "CARACTERÍSTICAS DE VENTO DA REGIÃO NORDESTE Análise, Modelagem e Aplicações para Projetos de Centrais Eólicas”, Tese de Mestrado em Engenharia Mecânica, Universidade Federal de Pernambuco

[9] ESTEVES, TERESA MARIA VELOSO NUNES SIMÕES (2004) "BASE DE DADOS DO POTENCIAL ENERGÉTICO DO VENTO EM PORTUGAL - METODOLOGIA E DESENVOLVIMENTO", Tese de Mestrado.Universidade de Lisboa. 